\title{
On Actual Maximum Exposure From 5G Multi-Column Radio Base Station Antennas
}

\author{
Bo Xu, Member, IEEE, Davide Colombi, Christer Törnevik, Member, IEEE, Fatemeh Ghasemifard, \\ and Jiajia Chen, Senior Member, IEEE
}

\begin{abstract}
The traditional approach of radio frequency electromagnetic field exposure compliance assessment is highly conservative when applied to radio base station antennas implementing beamforming. In this paper, an analytical model based on the queuing theory with a hyper-exponential service distribution time is developed to assess the time-averaged actual maximum exposure of 5G multi-column radio base station antennas by taking into account the effects of beam scanning over time. Using the measured antenna radiation patterns, the $5 \mathrm{G}$ downlink antenna precoding codebook, and assuming a conservative user equipment distribution, the ratio of the actual maximum exposure to the theoretical maximum exposure with $100 \%$ traffic load and 75\% time-division duplex downlink duty cycle is found to be less than 0.5 and 0.3 for four-transmitter and eight-transmitter radio base station antennas, respectively. These results show that assuming constant peak power transmission in a fixed direction leads to an overestimate of exposure also from conventional antennas characterized by only a few transmitters in addition to massive multi-input multi-output products.
\end{abstract}

Index Terms-5G, base station antenna, beamforming, electromagnetic field exposure.

\section{INTRODUCTION}

B EFORE radio base station (RBS) equipment is placed on the market and installed on a site, manufacturers and operators are normally required to perform radio frequency (RF) electromagnetic field (EMF) compliance assessments. The aim of EMF compliance assessments for RBS equipment is to determine so-called compliance boundaries or exclusion zones, outside of which the EMF exposure is below the relevant limits, such as those provided in [1], [2]. In practice, EMF exposure from RBS antennas serving wide range coverage is evaluated with the metric of incident power density in free space. The incident power density is well characterized by the antenna radiation patterns if the compliance boundaries are located in the antenna far-field region [3].

Traditionally, EMF compliance assessments of RBS equipment are conducted for the maximum power configuration without taking into consideration factors like variation of network traffic, scheduling time, and spatial distribution of served user equipment (UE). Although EMF exposure is supposed to be averaged over a certain period, typically 6 minutes, the traditional EMF compliance assessments for RBS do not

The work conducted by J. Chen was carried out with the support from Swedish Foundation of Strategic Research, and Swedish Research Council.

B. Xu, D. Colombi, F. Ghasemifard, and C. Törnevik are with Ericsson Research, Ericsson AB, 16480 Stockholm, Sweden. (e-mail: bo.xu@ericsson.com).

J. Chen is with the Department of Electrical Engineering, Chalmers University of Technology, Sweden (e-mail: jiajiac@chalmers.se). apply time-averaging, except for deterministic factors like the time-division duplex (TDD) downlink (DL) duty cycle. This is referred to as the theoretical maximum exposure condition in EMF compliance assessments [3], [4].

A differentiating feature between the 5G New Radio (NR) and the previous generations of cellular communication technologies is the beam-centric design, which aims to transmit energy in the directions where it is needed rather than to constantly transmit energy in a wide angular sector [5]-[8]. The effects of beam scanning constitute a stochastic factor that affects the time-averaged EMF exposure. If applying the theoretical maximum exposure condition, the maximum power would be assumed to be continuously transmitted in each possible direction, resulting in unrealistic and very conservative EMF exposure levels and compliance boundaries. To avoid overly conservative assessments, it has been specified in the international standard International Electrotechnical Commission (IEC) 62232:2017 [3] that EMF exposure assessments can be conducted for the actual maximum exposure condition corresponding, e.g., to the 95th percentile of all possible exposure scenarios. In an IEC technical report [4], two case studies based on [9] and [10] are provided on realistic exposure evaluations of $5 \mathrm{G}$ massive multi-input multi-output (MIMO) RBS. Recently, another theoretical model regarding actual maximum exposure of massive MIMO RBS using a statistical approach is published [11]. Numerical approaches that utilize the ray-tracing method to model actual maximum exposure of 5G massive MIMO RBS are also applied with realistic signal propagation conditions [11], [12]. In these studies, statistical models are developed to obtain a conservative but realistic EMF exposure for a significant proportion of all possible DL exposure scenarios. Recent EMF exposure measurements on massive MIMO RBSs [13]-[15] demonstrate that the realistic time-averaged exposure is well below the actual maximum exposure derived from the theoretical models [9]-[12].

Similar to massive MIMO RBSs, which typically have more than 16 transmitters (TXs) and enable dozens or even hundreds of beam realizations, the conventional multi-port RBSs with a few TXs, e.g., four or eight, can also perform beamforming with a limited number of beam realizations in NR. With smaller degrees of freedom in beam realizations, the difference between the actual and the theoretical maximum exposure for such RBS antennas is expected to be smaller compared to that for the massive MIMO RBS. In this paper, a systematical analysis is performed to characterize the actual maximum exposure, i.e., 95th percentile realistic time-averaged exposure, for 4TX and 8TX RBS antennas. The proposed model of the 
actual maximum exposure is built upon the antenna precoding matrix codebook provided in the 3rd Generation Partnership Project (3GPP) NR specification [16] and a queuing model with hyper-exponential service distribution for 6-minute EMF averaging time.

\section{Problem Formulation}

The beamforming methods applicable in NR are briefly discussed in the beginning of this section. Then, the problem of actual maximum exposure is formulated according to one of the NR beamforming strategies and the conservative assumptions made on the transmission scheme, and the implications of the evaluated actual maximum exposure are discussed. Afterwards, a queuing model is used to derive the analytical expression of actual maximum exposure.

\section{A. Beamforming in NR}

In $5 \mathrm{G}$ NR, DL beamforming can be performed through channel sounding supported by either the DL channel state information reference signals (CSI-RS) or uplink (UL) sounding reference signals (SRS). With CSI-RS beamforming, the UE makes use of CSI-RS transmitted by the RBS to identify the best possible available beam. A precoding matrix indicator (PMI) and a rank indicator (RI) are therefore sent by the UE in the channel state information (CSI) report back to the RBS. The PMI and RI contain the indices to the antenna precoding matrix from the codebook defined in the 3GPP NR specification [16], which is known by both RBS and UE. After receiving the CSI report, the RBS selects the beam(s) to be used for DL traffic data transmission based on the reported PMI and RI. With SRS beamforming, mainly used for TDD, the RBS utilizes the reciprocity of the DL and UL channels to create beams for transmission of DL traffic data using the SRS sent by UE. In this paper, the actual maximum exposure is assessed for CSI-RS beamforming. As implied in [11] and [12], the reciprocity-based beamforming in general leads to a lower actual maximum exposure compared to the codebookbased beamforming.

\section{B. Time-averaged EMF exposure}

In the far field, EMF exposure from RBS is well characterized by the antenna radiation pattern, because the power density is proportional to the antenna gain. For the theoretical maximum exposure condition, an EMF compliance assessment is conducted assuming peak power transmission for all possible beam realizations. In practice, the RBS steers the beams in several different wanted directions over the EMF averaging period. Therefore, the time-averaged exposure is far below the theoretical maximum. From the perspective of transmitted power, the time-averaged exposure in a particular direction can be considered as a fixed beam transmitting with a fraction of peak power in that direction. Thus, the problem to evaluate the actual maximum exposure can be transformed to assessing the ratio of the actual to the theoretical maximum exposure.

In the modern cellular network, the network traffic is highly bursty and swiftly switched between different UEs. To evaluate the statistical profiles of time-averaged exposure and also to facilitate the use of the proposed model, the EMF averaging period $T$ is equally divided into $M$ number of time intervals $\Delta t$. Considering the effects of beam scanning, the ratio of the time-averaged exposure to the theoretical maximum exposure over $T$ in the spherical coordinate system can be written as

$$
H(\theta, \phi)=\frac{1}{M} \sum_{s=1}^{M} h_{s}(\theta, \phi, \Delta t)
$$

where $h_{s}$ represents the ratio of time-averaged exposure to the theoretical maximum exposure during the $s$ th time interval $\Delta t$. If the RBS can form $c$ number of beam patterns and transmits assuming full buffer, $h_{s}$ can be further expressed as

$$
h_{s}(\theta, \phi, \Delta t)=\frac{f_{\mathrm{TDD}} \sum_{l=0}^{c-1} U(\theta, \phi, l) F_{s}(l, \Delta t)}{\sum_{l=0}^{c-1} F_{s}(l, \Delta t)},
$$

where $f_{\mathrm{TDD}}$ is the deterministic factor accounting for the TDD DL duty cycle, $F_{s}(l, \Delta t)$ is the energy transmitted through the $l$ th beam during $\Delta t$, and $U(\theta, \phi, l)$ denotes the normalized radiation gain. $U(\theta, \phi, l)$ can be written as the ratio of the $l$ th beam radiation gain to the radiation gain of the beam pattern envelope in the direction $(\theta, \phi)$

$$
U(\theta, \phi, l)=\frac{G(\theta, \phi, l)}{\max _{l=0,1, \ldots, c-1} G(\theta, \phi, l)}, \quad l=0,1, \ldots, c-1,
$$

where $G(\theta, \phi, l)$ is the gain of the $l$ th beam in the linear scale.

Assume that there are $N_{s}(\Delta t)$ number of 'data groups' (DGs) to be transmitted during $D t$ and the transmitted energy is equally divided between them. Here, the DG can be understood as a bundle of packet data that has to be transmitted to a single UE. Different DGs can be transmitted to the same UE or different UE during $\Delta t$. Denoting the number of DGs transmitted through the lth beam during the $s$ th $\Delta t$ by $n_{s}(l, \Delta t)$, we have $N_{s}(\Delta t)=\sum_{l=0}^{c-1} n_{s}(l, \Delta t)$, and (2) can be expressed as

$$
h_{s}(\theta, \phi, \Delta t)=\frac{f_{\mathrm{TDD}} \sum_{l=0}^{c-1} U(\theta, \phi, l) n_{s}(l, \Delta t)}{N_{s}(\Delta t)} .
$$

As defined in [9] and [11], the 95th percentile of $H(\theta, \phi)$, denoted $H_{0.95}(\theta, \phi)$, is conceived as the scaling factor between the actual and the theoretical maximum exposure. By considering the exposure scenario and relevant inputs in a very conservative way, the so-called power reduction factor (PRF) [4] can be determined from the maximum value of $H_{0.95}(\theta, \phi)$ over the scan range of the RBS antenna. In this way, the derived PRF can be multiplied by the maximum transmitted power from the RBS to determine the actual maximum exposure for similar antenna array configurations in other EMF compliance assessments.

To facilitate the evaluation of the actual maximum exposure using (2) and the queuing model described in the next subsection, we make the following conservative assumptions on the transmission scheme. An illustration of the transmission scheme according to these assumptions can be found in Fig. 1.

- The RBS utilization defined as the ratio of used DL resources to the available DL resources remains at full 


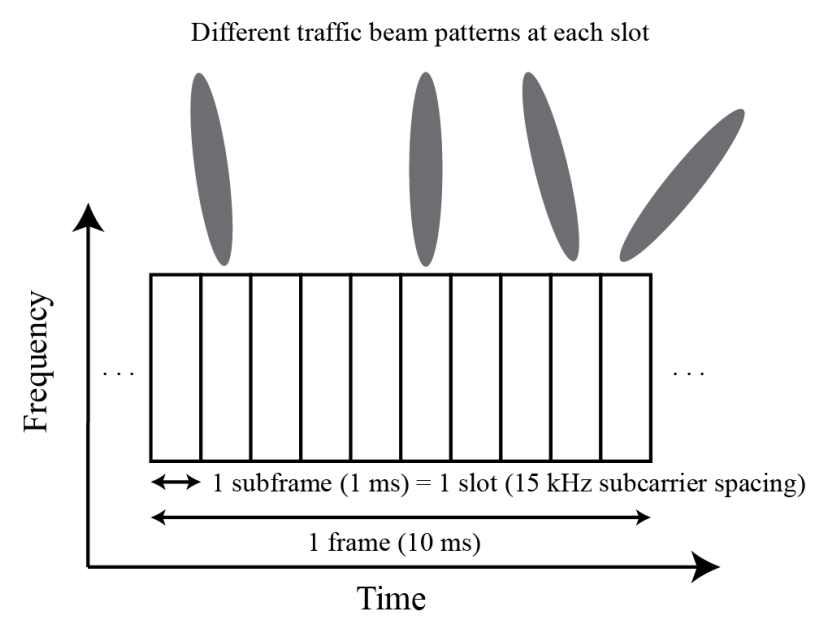

Fig. 1. Illustration of the NR frame structure with different 1-layer beams on different slots (no FDM).

buffer, i.e., $\rho=100 \%$. The TDD DL duty cycle $f_{\mathrm{TDD}}=$ $75 \%$.

- In the time domain, $\Delta t$ is chosen as the length of a radio frame, i.e., $10 \mathrm{~ms}$, consisting of 10 subframes of $1 \mathrm{~ms}$ each. In NR, the slot is the basic scheduling unit ${ }^{1}$. One subframe can contain at least 1 slot corresponding to $15 \mathrm{kHz}$ subcarrier spacing (SCS) and up to 32 slots corresponding to $480 \mathrm{kHz}$ SCS. From the EMF exposure point of view, a larger number of slots within the EMF exposure averaging period means a lower actual maximum exposure. Thus, in this paper, the $15 \mathrm{kHz}$ SCS, i.e., 10 slots per frame, is used.

- In the frequency domain, the RBS can allocate different physical resource blocks (PRBs) consisting of 12 subcarriers to different UE. Different PRBs can be allocated to different beams by means of frequency division multiplexing (FDM). However, this means the energy is more frequently spread to other directions, resulting in a lower exposure level. Thus, we do not consider FDM but time division multiplexing (TDM).

- In the spatial domain, the RBS may choose to simultaneously transmit energy in several directions and different polarizations associated with different MIMO layers to maximize data throughput. From an EMF exposure assessment point of view, the maximum exposure is typically obtained when a focused beam with only one main lobe is used. Thus, we assume 1-layer beamforming in the spatial domain.

- The broadcast and control channels are neglected in the model, as energy they carry is negligible in NR compared to traffic data.

\section{Queuing model}

The schematic view of the queuing model denoted by $\mathrm{M} / \mathrm{Hc} / 1$ [17], is shown in Fig. 2. In this model, both the arrival

\footnotetext{
${ }^{1} \mathrm{NR}$ also supports the so-called mini-slot [6] that allows transmission at any symbol within a slot, but it mainly aims for latency-critical mission, licensed assisted access, and transmission in the millimeter-wave spectrum.
}

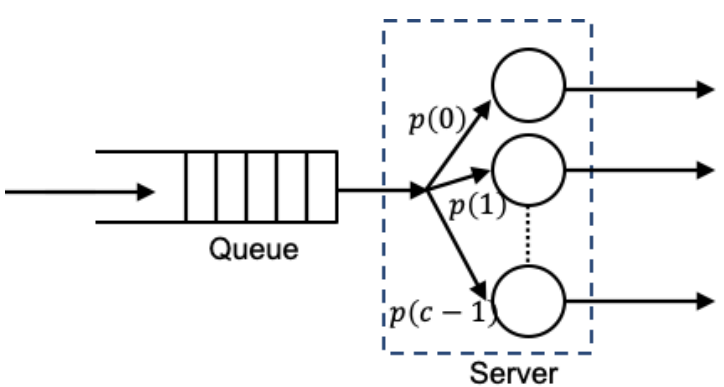

Fig. 2. Illustration of $\mathrm{M} / \mathrm{Hc} / 1$ queuing model having $c$ options in one server. Each option corresponds to one 1-layer CSI-RS beam.

of users and the number of users served with each option of the server follow the Poisson process. The server has $c$ options in total, leading to a hyper-exponentially distributed service time. The users are served one by one by selecting one option in the server, and there is no correlation between different options. In this paper, the user is a term only used in the queuing model, and it is not interchangeable with UE.

We can draw an analogy between this queuing model and the RBS transmitting the 1-layer CSI-RS beamforming in the following way:

- The options to serve the users are the 1-layer CSI-RS beams, and the $l$ th option corresponds to the $l$ th beam.

- The system utilization $\rho=\lambda / \mu$ in the static state of the queuing model is analogues to the RBS utilization, in which $\lambda$ is the arrival rate, and $\mu$ is the service rate. $\lambda$ and $\mu$ are constant for the static state

- One user corresponds to one DG, for which the power contribution in the direction $(\theta, \phi)$ is weighted by $U(\theta, \phi, l)$. The number of users served during one $\Delta t$ by the $l$ th option is analogous to the number of DGs transmitted through the $l$ th beam during that $\Delta t$, i.e., $n_{s}(l, \Delta t)$.

- The probability of a user from the waiting area to be served by the $l$ th option corresponds to the probability of a DG to be transmitted to a single UE through the $l$ th beam, and it is denoted by $p(l)$. Note $\sum_{l=0}^{c-1} p(l)=1$.

As we assume that each option offers the service with the same average service time $\mu \Delta t$ and there is no correlation between options, i.e., the $n_{s}(l, \Delta t)$ is independent for different $l$, the probability of serving $K$ users during one $\Delta t$ follows a Poisson distribution

$$
\operatorname{Pr}\left(N_{s}(\Delta t)=K\right)=\frac{(\mu \Delta t)^{K} e^{-\mu \Delta t}}{K !}, \quad K=0,1, \ldots
$$

For a given $N_{s}(\Delta t)=K$, the probability of the combination $\left\{n_{s}(l, \Delta t)=k(l), l=0,1, \ldots, c-1 \mid N_{s}(\Delta t)=K\right\}$ for $K=\sum_{l=0}^{c-1} k(l)$ follows a multinomial distribution according to the property of Poisson distributions [18]

$$
\begin{aligned}
\operatorname{Pr} & \left\{n_{s}(l, \Delta t)=k(l) \mid N_{s}(\Delta t)=K\right\} \\
& \sim \operatorname{Multinom}(K,\{p(l)\}), \quad l=0,1, \ldots, c-1 .
\end{aligned}
$$

As the number of transmitted DGs during one $\Delta t$ and the next $\Delta t$ are assumed independent, i.e., $N_{s}(l, \Delta t)$ is independent for different $s, h_{s}(\theta, \phi, \Delta t)$ is also independent for different $s$ in this model. As $h_{s}(\theta, \phi, \Delta t)$ is not 
TABLE I

Multi-Column Antennas Used In This Paper

\begin{tabular}{c|c|c|c}
\hline \hline Antenna & Type & $\begin{array}{c}\text { Electrical } \\
\text { Downtilt }\end{array}$ & Frequency Span \\
\hline \hline $\begin{array}{c}\text { Ericsson/Kathrein } \\
80010902\end{array}$ & $4 \mathrm{TX}$ & $1 \mathrm{deg}$ & $698-960 \mathrm{MHz}$ \\
\hline $\begin{array}{c}\text { Ericsson/Kathrein } \\
80010652\end{array}$ & $4 \mathrm{TX}$ & $0 \mathrm{deg}$ & $1710-2690 \mathrm{MHz}$ \\
\hline $\begin{array}{c}\text { Ericsson/Kathrein } \\
80020622\end{array}$ & $4 \mathrm{TX}$ & $2 \mathrm{deg}$ & $1710-2690 \mathrm{MHz}$ \\
\hline $\begin{array}{c}\text { Ericsson/Kathrein } \\
80010922\end{array}$ & $4 \mathrm{TX}$ & $2 \mathrm{deg}$ & $3300-3800 \mathrm{MHz}$ \\
\hline $\begin{array}{c}\text { Ericsson/Kathrein } \\
800250911\end{array}$ & $8 \mathrm{TX}$ & $3 \mathrm{deg}$ & $3300-3800 \mathrm{MHz}$ \\
\hline \hline
\end{tabular}

only independent but also identically distributed, according to the Lindeberg-Lévy central limit theorem, the sum of $h_{s}(\theta, \phi, \Delta t)$, i.e., $H(\theta, \phi)$, follows the normal distribution [18]

$$
H(\theta, \phi) \sim \mathcal{N}\left(\mu_{h}(\theta, \phi), \frac{\sigma_{h}^{2}(\theta, \phi)}{M}\right),
$$

where $\mu_{h}(\theta, \phi)$ and $\sigma_{h}^{2}(\theta, \phi)$ are the mean and variance of $h_{s}(\theta, \phi, \Delta t)$. Therefore, the 95th percentile of $H(\theta, \phi)$ is

$$
H_{0.95}(\theta, \phi)=\frac{z_{0.95} \sigma_{h}(\theta, \phi)}{\sqrt{M}}+\mu_{h}(\theta, \phi),
$$

where $z_{0.95} \approx 1.6449$ is the 95 th percentile of a standard normal distribution.

\section{Model InPut Selection}

In this section, the characteristics of typical 4TX and 8TX RBS antennas currently available on the market are presented, and the antenna precoding matrix for 1-layer CSI-RS beamforming is determined according to [16]. The UE distribution generating the highest realistic exposure in [9] is used to compute the service probability $p(l)$ of each beam, leading to a conservative assessment of $H_{0.95}(\theta, \phi)$. At last, the parameters related to the queuing model are chosen in a way to obtain the conservative $H_{0.95}(\theta, \phi)$.

\section{A. RBS Antennas}

Commercially available RBS antennas are used to evaluate the actual maximum exposure (see Table I). The measured embedded antenna patterns are provided over the operating frequency span for each port with lowest electrical downtilt angle by the development unit of Ericsson. The electrical downtilt is widely used in RBS antennas to keep the main lobe of the radiation pattern below the azimuthal plane.

\section{B. Antenna Precoding Weights}

A schematic view of the port convention for horizontally arranged 4TX and 8TX RBS antennas is shown in Fig. 3. The antenna precoding matrix for 1-layer CSI reporting can be written as [16]

$$
W_{l, n}=\frac{1}{\sqrt{N_{\mathrm{CSI}-\mathrm{RS}}}}\left[\begin{array}{c}
\nu_{l} \\
\varphi_{n} \nu_{l}
\end{array}\right],
$$

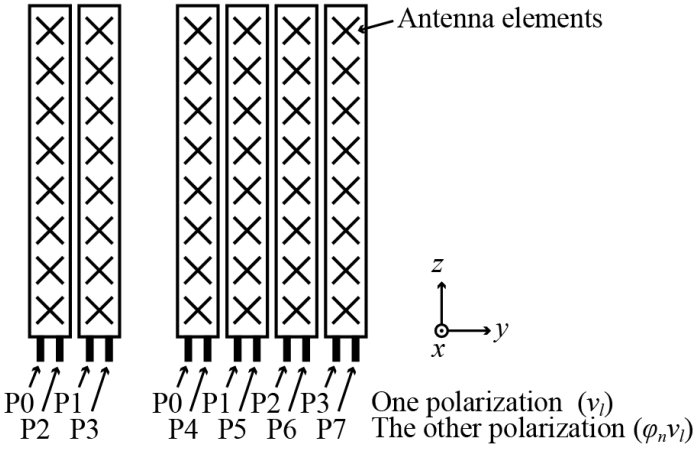

Fig. 3. Schematic view of port convention for horizontally arranged 4TX and 8TX antennas considered in this paper.

TABLE II

PARAMETERS OF 1-LAYER CSI REPORTING CODEBOOK FOR Horizontally ARRANGEd MUlTi-COLUMN ANTENNAS

\begin{tabular}{c|c|c|c}
\hline \hline Type & $N_{\text {CSI-RS }}$ & $N_{1}$ & $c$ \\
\hline \hline 4TX & 4 & 2 & 8 \\
\hline 8TX & 8 & 4 & 16 \\
\hline \hline
\end{tabular}

where

$$
\begin{aligned}
& \nu_{l}=\left[1, e^{j 2 \pi l / c}, \ldots, e^{j 2 \pi l\left(N_{1}-1\right) / c}\right]^{\mathrm{T}}, l=0,1, \ldots, c-1, \\
& \varphi_{n}=e^{j \pi n / 2}, \quad n=0,1,2,3,
\end{aligned}
$$

$N_{\text {CSI-RS }}$ is the number of CSI-RS antenna ports, $N_{1}$ is a parameter specified by the 3GPP depending on the CSI-RS configuration. The values of $N_{\mathrm{CSI}-\mathrm{RS}}, N_{1}$, and $c$ for horizontally arranged 4TX and 8TX antennas are provided in Table II. Note that $\nu_{l}$ and $\varphi_{n} \nu_{l}$ are the antenna precoding vectors for the ports of different polarizations (see Fig. 3). The subscript $n$ indicates the polarization of the field, yet has no impact on the analysis of this work. Thus for simplicity $n=0$ is assumed, meaning that, for example for 4TX antennas, the excitation weights of P0 and P1 is identical to the weights of $\mathrm{P} 2$ and $\mathrm{P} 3$, respectively. The 1-layer CSI-RS beam radiation patterns are obtained by applying (9) to the measured pattern per port of the provided antennas.

\section{Service Probability}

At each angle within the scan range, the beam with the maximum gain is selected from the codebook to establish service to UE. Using the established CSI-RS beam radiation patterns, the angular service establishment range $\left(\hat{\theta}_{l}, \hat{\phi}_{l}\right)$ can be specified as

$$
\begin{array}{r}
\left(\hat{\theta}_{l}, \hat{\phi}_{l}\right)=\{(\theta, \phi) \mid G(\theta, \phi, l)>G(\theta, \phi, m), l \neq m\}, \\
l, m=0,1, \ldots, c-1 .
\end{array}
$$

The spatial distribution of UE served by a 5G RBS will impact actual maximum exposure as it determines how the beams are steered. For a typical RBS covering $120^{\circ}$ in azimuth and $30^{\circ}$ in elevation, four UE distribution scenarios are defined in [9] for the evaluation of actual maximum exposure. The UE distribution depicted by a cosine function in azimuth and without elevation scanning gives the highest actual maximum 


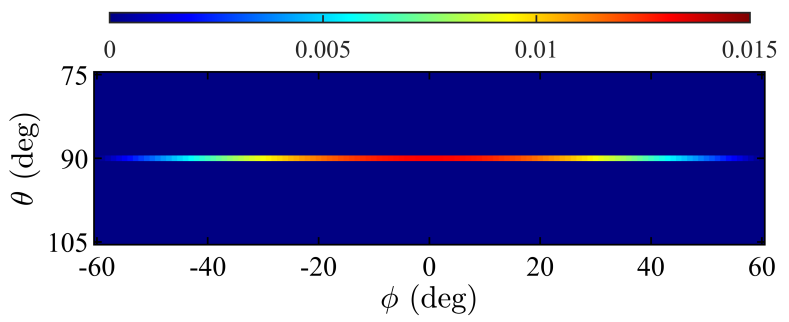

Fig. 4. One-dimensional UE distribution weighted by the cosine function for $\theta_{0}=90^{\circ}$. In the figure, the probability distribution function (11) is discretized and then normalized.

exposure levels in [9] for the massive MIMO RBS as this scenario has a more concentrated UE distribution density in the broadside beam direction. Thus, we choose this UE distribution here for the 4TX and 8TX antennas. This spatial UE distribution density can be expressed as

$$
w(\theta, \phi)= \begin{cases}\frac{3}{4} \delta\left(\theta-\theta_{0}\right) \cos \frac{3 \phi}{2}, & -60^{\circ} \leq \phi \leq 60^{\circ} \\ 0, & \text { otherwise }\end{cases}
$$

where $\theta_{0}$ is the zenith angle for the electrical downtilt. Fig. 4 shows the spatial UE distribution density over the scan range. Using the angular service establishment range (10) and the spatial UE distribution density (11), the probability of a single UE to be served by the $l$ th beam can be expressed as

$$
p(l)=\int_{\hat{\theta}_{l}} \int_{\hat{\phi}_{l}} w(\theta, \phi) \sin \theta \mathrm{d} \theta \mathrm{d} \phi, l=0,1, \ldots, c-1 .
$$

\section{Parameters in Queuing Model}

The $H_{0.95}(\theta, \phi)$ relies on the property of the probability mass function of $h_{s}(\theta, \phi, \Delta t)$ and the value of $M$. To make a conservative assessment of actual maximum exposure, the following parameters are selected in the queuing model:

- The parameter $\Delta t$ has been chosen to be $10 \mathrm{~ms}$, i.e., 1 frame including 10 slots $(15 \mathrm{kHz}$ SCS). For TDM, this means the number of active UEs within $\Delta t$ is less than or equal to 10 . Thus, $K=$ $1,2, \ldots, 10$. By enumerating all these possible combinations of $\left\{n_{s}(l, \Delta t), l=0,1, \ldots, c-1\right\}$ in (6) for $K=1,2, \ldots, 10$, the terms $\mu_{h}(\theta, \phi), \sigma_{h}^{2}(\theta, \phi)$, and thus (8) can be calculated numerically. For an EMF exposure averaging period of $T=6$ minutes, $M=T / \Delta t=$ 36000 .

- The parameter $\mu \Delta t$ is the mean value of the Poisson distribution (5). Since $K=1,2, \ldots, 10$, the range of $\mu \Delta t$ must be chosen within $[1,10]$. In addition, a smaller $\mu \Delta t$ means the RBS statistically sends fewer DGs, and this will naturally lead to a more conservative, i.e., higher, $H_{0.95}$. Therefore, we choose $\mu \Delta t=1$.

\section{RESUlts}

In this section, firstly the beam radiation patterns and service probabilities of the 4TX antenna Ericsson/Kathrein 80020622 and the 8TX antenna Ericsson/Kathrein 800250911 are illustrated (Figs. 5--8) and discussed. Then, the obtained
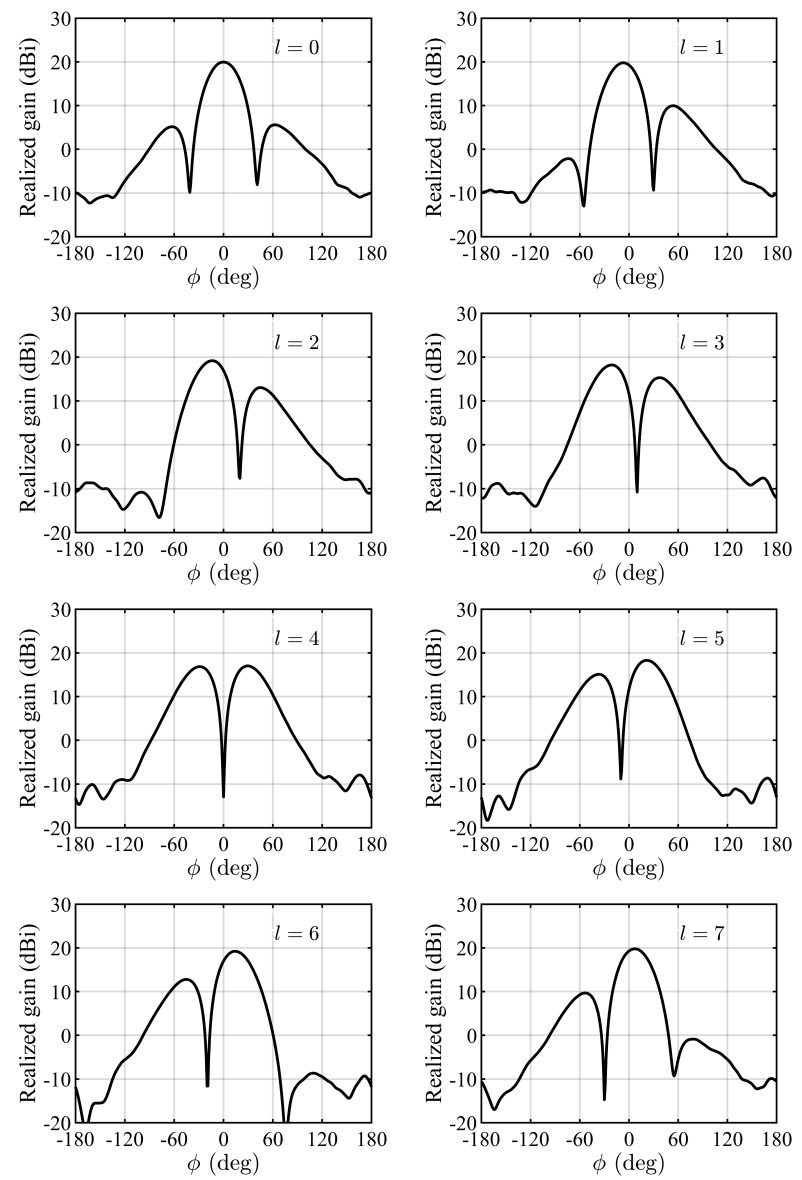

Fig. 5. Measured 1-layer CSI-RS beam radiation patterns for the 4TX antenna (Ericsson/Kathrein 80020622) at $1710 \mathrm{MHz}$ and $\theta=\theta_{0}=92^{\circ}$.

$H_{0.95}$ values for all the antennas listed in Table I over their frequency span at broadside direction and over the azimuthal scanning angle at their lowest frequency are depicted (Fig. 9 and Fig. 10).

Fig. 5 shows the 1-layer CSI-RS beam radiation patterns for the 4TX antenna at $1710 \mathrm{MHz}$. The main lobe of the beams is scanned from $\phi=0^{\circ}$ to $\phi=-60^{\circ}$ for $l$ from 0 to 5 , and is scanned from $\phi=60^{\circ}$ to $\phi=0^{\circ}$ for $l$ from 3 to 7 and then back to $l=0$. Note that the maximum gain at large scan angles is provided by the side lobes of the beams. The CSI-RS beam radiation patterns of the $8 \mathrm{TX}$ antenna have more fine-grained beams yet not shown here for simplicity.

Using the obtained beam radiation patterns, $U(\theta, \phi, l)$ observed in the broadside beam direction $\left(\theta=\theta_{0}, \phi=0^{\circ}\right)$ for the mentioned 4TX and 8TX antennas are shown in Fig. 6. The power contribution in the observation direction is mostly from the beams with maximum gain close to the observation direction. For the 4TX antenna, the beams with maxima far from the observation direction still have some contributions to the observation direction, while for the 8TX antenna, only the closest beams have major contributions. Such behavior is not only valid for the broadside, but also true when observing in other directions. This is one of the key factors that differentiates the $H_{0.95}$ levels between 4TX and 8TX antennas in Fig. 9 and Fig. 10. 


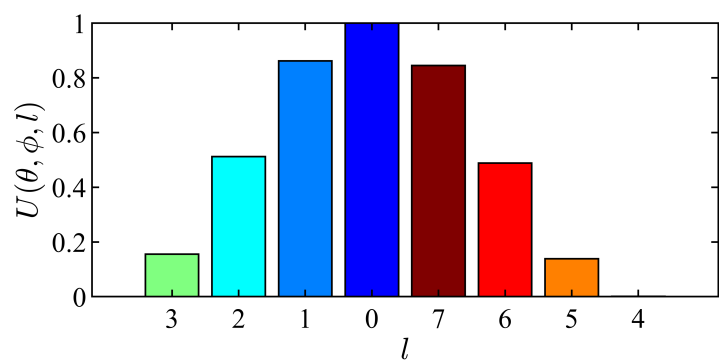

(a)

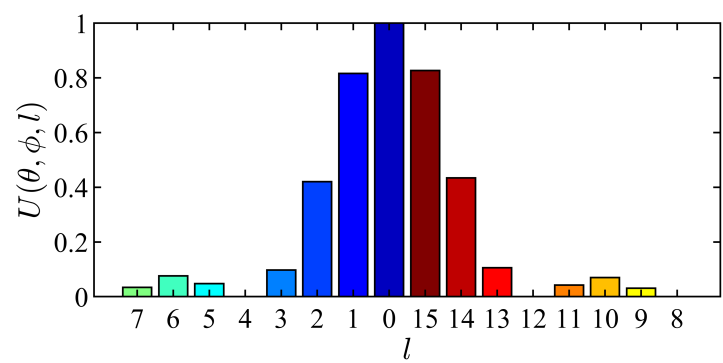

(b)

Fig. 6. Normalized power fraction of each CSI-RS beam contributed to the broadside beam direction. (a) Ericsson/Kathrein 80020622 at $1710 \mathrm{MHz}$ $\left(\theta_{0}=92^{\circ}, \phi=0^{\circ}\right)$, (b) Ericsson/Kathrein 800250911 at $3300 \mathrm{MHz}$ $\left(\theta_{0}=93^{\circ}, \phi=0^{\circ}\right)$.

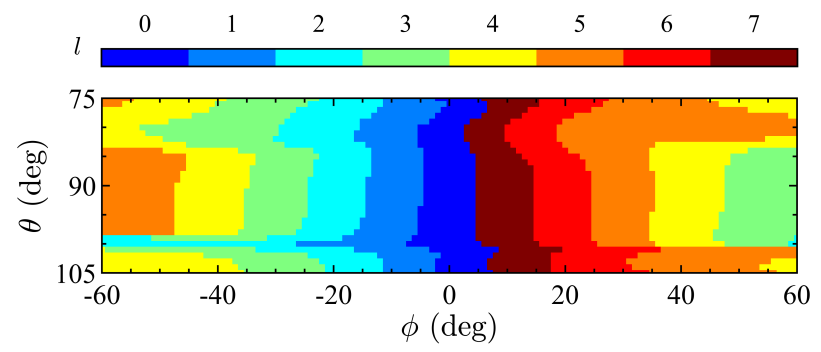

(a)

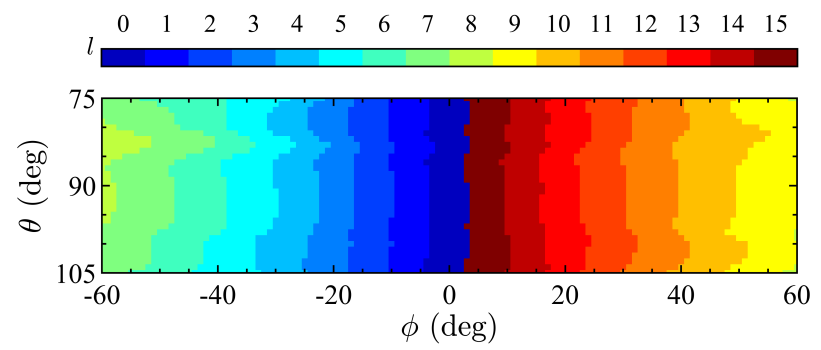

(b)

Fig. 7. Angular range for each CSI-RS beam establishing service in the range $-60^{\circ} \leq \phi \leq 60^{\circ}$ and $75^{\circ} \leq \theta \leq 105^{\circ}$ for (a) 4TX antenna Ericsson/Kathrein 80020622 at $1710 \mathrm{MHz}$, (b) 8TX antenna Ericsson/Kathrein 800250911 at $3300 \mathrm{MHz}$.

Fig. 7 depicts the angular service establishment range for the 1-layer CSI-RS beams of the mentioned 4TX antenna at $1710 \mathrm{MHz}$ and the 8TX antenna at $3300 \mathrm{MHz}$. It can be observed that the beams close to the broadside have smaller angular coverage than the beams close to the edge of the scan range. This phenomenon leads to a smooth variation in $p(l)$ even when the UE distribution has its maximum in the

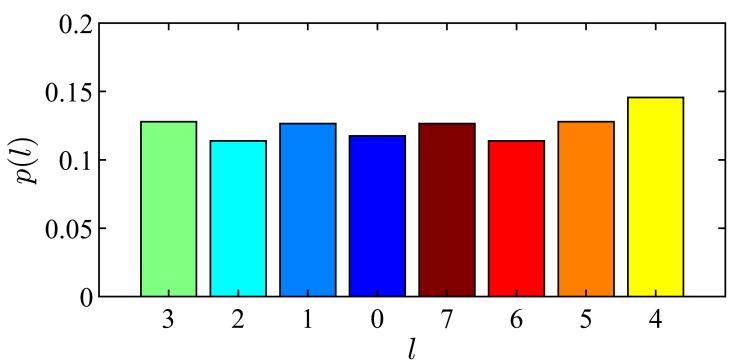

(a)

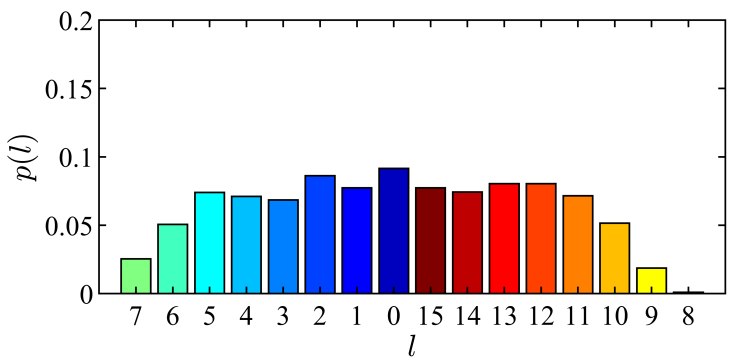

(b)

Fig. 8. Probability mass function of service establishment by each CSI-RS beam of (a) 4TX antenna Ericsson/Kathrein 80020622 at $1710 \mathrm{MHz}$, (b) 8TX antenna Ericsson/Kathrein 800250911 at $3300 \mathrm{MHz}$.

broadside direction. As can be seen from Fig. 8, using the onedimensional cosine UE distribution (11), the probabilities of serving UE by each CSI-RS beam by the mentioned antennas are relatively smooth over the scan range, particularly for the 4TX antenna.

Fig. 9 shows the $H_{0.95}$ values in the broadside beam direction $\left(\theta=\theta_{0}, \phi=0^{\circ}\right)$ for the provided antennas over their frequency spans. The maximum $H_{0.95}$ values are 0.44 and 0.24 for the $4 \mathrm{TX}$ and $8 \mathrm{TX}$ antennas, respectively. The difference between the 4TX and 8TX antennas is mostly due to $U(\theta, \phi, l)$, as mentioned above for Fig. 6. In Fig. 10, the $H_{0.95}$ values for $\theta=\theta_{0}$ and different $\phi$ are presented at the lowest operating frequencies. For the 4TX and 8TX antennas, the $H_{0.95}$ values range from 0.33 to 0.45 and from 0.10 to 0.25 , respectively. Note that the variation of $H_{0.95}$ for the 8TX antenna looks similar to the maximum gain at different directions, however, the variation of $H_{0.95}$ for the 4TX antennas is relatively smooth. This can be attributed to the distributions of both $U(\theta, \phi, l)$ and $p(l)$, for which $U(\theta, \phi, l)$ and $p(l)$ are more concentrated in the broadside beam direction for the $8 \mathrm{TX}$ antenna, while smoother over the scan range for the $4 \mathrm{TX}$ antennas.

\section{DISCUSSION}

The proposed model considers neither the multi-layer MIMO nor the sharing of frequency resources between different UEs (i.e., do not consider FDM). Heuristically, the use of FDM can be seen to have a larger range for $N_{s}(\Delta t)$ in (4), leading to a lower (i.e., less conservative) $H_{0.95}$. The use of multi-layer MIMO can be considered as a superposition of a greater number of one-beam transmissions with each beam carrying less power. This implies an increasing $M$, also 


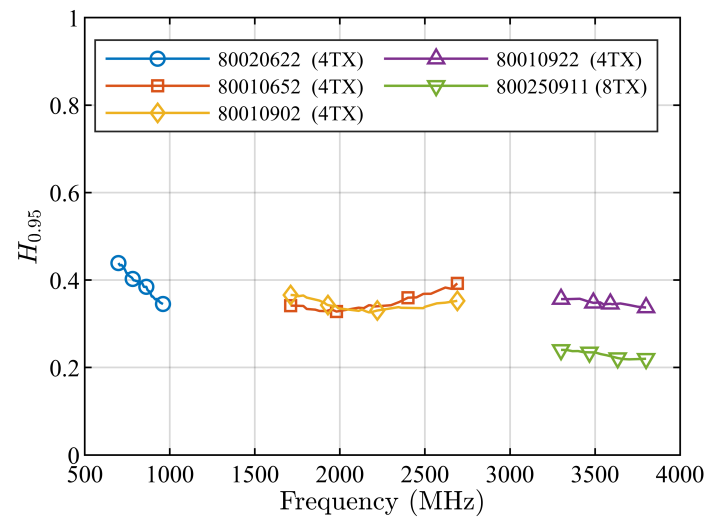

Fig. 9. $H_{0.95}$ at the broadside direction $\left(\theta=\theta_{0}, \phi=0^{\circ}\right)$ for investigated antennas over their frequency spans. The results are given for $\rho=100 \%$ and $f_{\mathrm{TDD}}=75 \%$.

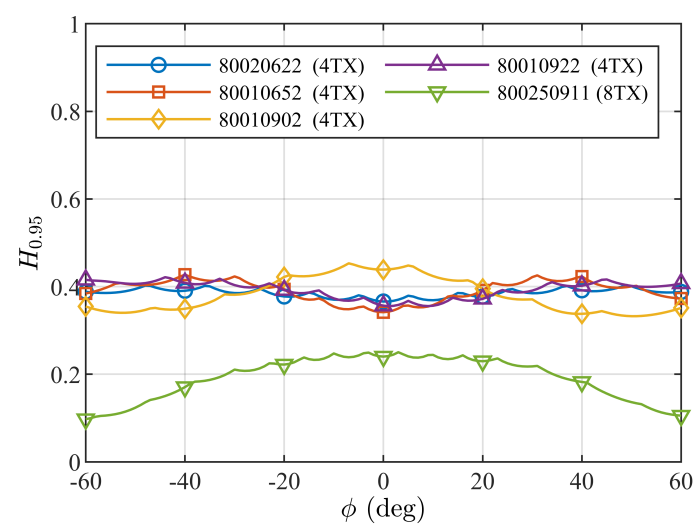

Fig. 10. $H_{0.95}$ for the investigated antennas at their lowest operating frequencies with different $\phi$ at the electrical downtilt $\theta=\theta_{0}$. The results are given for $\rho=100 \%$ and $f_{\mathrm{TDD}}=75 \%$.

leading to lower $H_{0.95}$. This is also partly supported by [11] and [12], in which the conclusions are given by means of Monte Carlo analyses.

The model is built upon the Poisson process, for which the arrivals at one $\Delta t$ is independent of another $\Delta t$. This model can make good predictions when the RBS utilization is relatively high and the number of active UEs is relatively large. Under this condition, the transmitted DGs can be swiftly allocated from one UE to another UE. However, when the RBS utilization is very low and the number of active UE is very small, the probability of transmitting different DGs to one UE is higher. In such a case, some other arrival process models may better depict the 95th percentile realistic time-averaged exposure. Nevertheless, the actual maximum exposure under the high RBS utilization is typically higher than the actual maximum exposure under low RBS utilization. In other words, the $H_{0.95}$ value derived from a very low RBS utilization is very unlikely to exceed that derived from a high RBS utilization [4]. Considering the results presented in Fig. 9 and Fig. 10 for different frequencies, different angles, full buffer $(\rho=100 \%)$, and together with other conservative assumptions, the PRF values of 0.5 and 0.3 can be recommended for $4 \mathrm{TX}$ and $8 \mathrm{TX}$
NR RBS antennas with $f_{\text {TDD }}=75 \%$. For other $f_{\text {TDD }}$ values, for example, $60 \%$, the recommend PRF values can be scaled from 0.5 to $0.5 / 0.75 \times 0.6=0.4$ for $4 \mathrm{TX}$ antennas.

It is worth noting that in [12], the ideal $2 \times 2$ dipole array (2column) leads to a very conservative 95 th percentile of timeaveraged exposure in the ray-tracing simulation, because the generated beams are not directive, as explained in [12]. In this work, by use of commercial 4TX RBS antennas with beam gain greater than $20 \mathrm{dBi}$, it is clearly shown that the actual maximum exposure for 4TX antennas (2-column) is well below the theoretical maximum. For the 8TX antenna (4column), the levels of actual maximum exposure agree well with the ray-tracing simulation results in [12] with the $4 \times 4$ array (4-column).

The $H_{0.95}$ values derived above are for RBS scanning only in azimuthal plane. For NR RBS antennas with both horizontally and vertically arranged ports enabling beamforming in both directions, the extra degrees of freedom naturally results in lower $H_{0.95}$ levels compared to the antennas with the same number of columns that scan only in the azimuthal plane ${ }^{2}$.

For EMF compliance assessment of classical multi-column antennas, the envelope of traffic beam radiation patterns can also be obtained by summing the antenna radiation pattern per port in a correlated way [19]-[22]. This is done by assuming the far-field strength of each port in phase over the sphere and combining them. As this approach gives the upper bound of EMF exposure for all possible beam realizations, applying the PRF to the combined exposure would also give the actual maximum exposure assessment with an extra margin.

\section{CONCLUSION}

In this paper, the actual maximum EMF exposure from 4TX and 8TX RBS antennas has been evaluated using the measured antenna patterns, the beamforming codebook specified in NR, a queuing model, and a cosine-shaped spatial UE distribution. With many conservative assumptions made in the proposed model, the ratios of the actual maximum to the theoretical maximum EMF exposure are found to be below 0.5 and 0.3 , respectively, for typical $4 \mathrm{TX}$ and $8 \mathrm{TX}$ RBS antennas, considering a 75\% TDD DL duty cycle. The results show the importance of considering the effect of beamforming when assessing actual maximum exposure also for conventional antennas characterized by fewer TXs in addition to massive MIMO products.

\section{ACKNOWLEDGMENT}

The authors would like to thank Robert Stensson, Jürgen Rumold, Anton Huber, and Soeren Konzack at Ericsson for the support with antenna measurements.

\footnotetext{
${ }^{2}$ This implies that, for example using the 3GPP terminology [16], the recommended PRF value for 4TX with CSI-RS configuration $\left(N_{1}=2\right.$, $N_{2}=1$ ) can also be applied to the RBS antennas with CSI-RS configuration $\left(N_{1}=2, N_{2}=2\right)$ without losing conservativeness, where $N_{2}>1$ accounts for the beam scanning in elevation similar to $N_{1}$ in (9). See [16] for the full description of CSI-RS beamforming.
} 


\section{REFERENCES}

[1] IEEE Standard for Safety Levels with Respect to Human Exposure to Electric, Magnetic, and Electromagnetic Fields, $0 \mathrm{~Hz}$ to $300 \mathrm{GHz}$, IEEE Std. C95.1, Oct. 2019.

[2] International Commission on Non-Ionizing Radiation Protection (ICNIRP), "Guidelines for limiting exposure to electromagnetic fields (100 kHz to $300 \mathrm{GHz}$ )," Health Phys., vol. 118, no. 5, pp. 483-524, May 2020.

[3] Determination of RF field strength, power density and SAR in the vicinity of radiocommunication base stations for the purpose of evaluating human exposure, International Electrotechnical Commission (IEC) Std. 62 232, Jun. 2017, Edition 2.0.

[4] "Case studies supporting IEC 62232 - determination of RF field strength and SAR in the vicinity of radiocommunication base stations for the purpose of evaluation human exposure," International Electrotechnical Commission (IEC), Tech. Rep. 62269, Apr. 2019, Edition 2.0.

[5] E. G. Larsson, O. Edfors, F. Tufvesson, and T. L. Marzetta, "Massive MIMO for next generation wireless systems," IEEE Commun. Mag., vol. 52, no. 2, pp. 186-195, Feb. 2014.

[6] E. Dahlman, S. Parkvall, and J. Sköld, 5G NR: The next generation wireless access technology, 2nd ed. Academic Press, 2020.

[7] H. Asplund et al., Advanced Antenna Systems for 5G Network Deployments: Bridging the Gap Between Theory and Practice. Academic Press, 2020.

[8] A. Osseiran, S. Parkvall, P. Persson, A. Zaidi, S. Magnusson, and K. Balachandran, "5G wireless access: an overview," white paper, Ericsson, Apr. 2020, 1/28423-FGB1010937.

[9] B. Thors, A. Furuskär, D. Colombi, and C. Törnevik, "Time-averaged realistic maximum power levels for the assessment of radio frequency exposure for 5G radio base stations using massive MIMO," IEEE Access, vol. 5, pp. 19711-19719, Sep. 2017.

[10] P. Baracca, A. Weber, T. Wild, and C. Grangeat, "A statistical approach for RF exposure compliance boundary assessment in massive MIMO systems," in WSA 2018; 22nd International ITG Workshop on Smart Antennas, 2018, pp. 1-6.

[11] D. Pinchera, M. Migliore, and F. Schettino, "Compliance boundaries of 5G massive MIMO radio base stations: A statistical approach," IEEE Access, vol. 8, pp. 182 787-182 800, Oct. 2020.

[12] S. Shikhantsov et al., "Ray-tracing-based numerical assessment of the spatiotemporal duty cycle of $5 \mathrm{G}$ massive MIMO in an outdoor urban environment," Appl. Sci., vol. 10, no. 21, p. 7631, Oct. 2020.

[13] D. Colombi et al., "Assessment of actual maximum RF EMF exposure from radio base stations with massive MIMO antennas," in 2019 PhotonIcs Electromagnetics Research Symposium - Spring (PIERS-Spring), 2019, pp. 570-577.

[14] R. Werner, P. Knipe, and S. Iskra, "A comparison between measured and computed assessments of the RF exposure compliance boundary of an in-situ radio base station massive MIMO antenna," IEEE Access, vol. 7 , pp. $170682-170689$, Nov. 2019.

[15] D. Colombi, P. Joshi, B. Xu, F. Ghasemifard, V. Narasaraju, and C. Törnevik, "Analysis of the actual power and EMF exposure from base stations in a commercial 5G network," Appl. Sci., vol. 10, no. 15, p. 5280, Jul. 2020.

[16] Physical layer procedures for data, 3rd Generation Partnership Project (3GPP) TS 38.214, Oct. 2020, Release 15.11.0.

[17] W. J. Stewart, "Queues with phase-type laws: Neuts' matrix-geometric method," in Probability, Markov Chains, Queues, and Simulation. Princeton University Press, 2009, ch. 12.

[18] — "Continuous distributions," in Probability, Markov Chains, Queues, and Simulation. Princeton University Press, 2009, ch. 7.

[19] "Guidance for evaluating exposure from multiple electromagnetic sources," International Electrotechnical Commission (IEC), Tech. Rep. 62630, Oct. 2010

[20] N. Perentos, S. Iskra, A. Faraone, R. J. McKenzie, G. Bit-Babik, and V. Anderson, "Exposure compliance methodologies for multiple input multiple output (MIMO) enabled networks and terminals," IEEE Trans. Antennas Propag., vol. 60, no. 2, pp. 644-653, Feb. 2012.

[21] B. Thors et al., "Radio frequency electromagnetic field compliance assessment of multi-band and MIMO equipped radio base stations," Bioelectromagnetics, vol. 35, no. 4, pp. 296-308, May 2014.

[22] B. Xu, M. Gustafsson, S. Shi, K. Zhao, Z. Ying, and S. He, "Radio frequency exposure compliance of multiple antennas for cellular equipment based on semidefinite relaxation," IEEE Trans. Electromagn. Compat., vol. 61, no. 2, pp. 327-336, Apr. 2019. 